\title{
Rendimento de grãos da soja em função do arranjo de plantas
}

\author{
Soybean yield response to plant arrangement
}

\section{Lisandro Rambo $^{1}$ José Antonio Costa $^{2}$ João Leonardo Fernandes Pires ${ }^{3}$ Geovano Parcianello $^{4}$ Felipe Gutheil Ferreira ${ }^{5}$}

\section{RESUMO}

A identificação do arranjo de plantas que resulte em menor competição intraespecifica permite melhor aproveitamento dos recursos disponiveis para o crescimento e rendimento de grãos da soja. $O$ experimento foi conduzido na Estação Experimental Agronômica da UFRGS em Eldorado do Sul, RS, na estação de crescimento 2000/01, objetivando avaliar como o arranjo de plantas de soja modifica a competição intraespecifica e de que forma isto se reflete no rendimento de grãos e seus componentes. Utilizou-se a cultivar 'BRS 137' (semiprecoce, determinada) em semeadura direta. $O$ delineamento experimental foi de blocos casualizados com parcelas sub-subdivididas e quatro repetições. Os tratamentos constaram de dois regimes hídricos (irrigado e não irrigado); dois espaçamentos entre linhas $(20$ e $40 \mathrm{~cm})$, e três populações de plantas $\left(20,30\right.$ e 40 plantas. $\left.m^{-2}\right)$. O rendimento de grãos foi afetado pela irrigação e pela interação espaçamento e população. O tratamento irrigado (5015 $\left.\mathrm{kg}^{\mathrm{h}} \mathrm{ha}^{-1}\right)$ apresentou

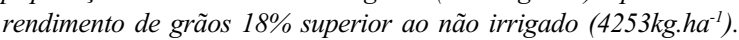
A maior taxa de enchimento de grãos (TEG), que resultou no maior peso do grão, explica o maior rendimento obtido no tratamento irrigado. $O$ arranjo de plantas que proporcionou maior rendimento de grãos foi a associação do espaçamento de $20 \mathrm{~cm}$ com a população de 20 plantas. $\mathrm{m}^{-2}$, obtendo-se $5014 \mathrm{~kg} . \mathrm{ha}^{-1} \mathrm{em}$ comparação a $4322 \mathrm{~kg} . \mathrm{ha}^{-1} \mathrm{em} 40 \mathrm{~cm}$ de espaçamento. Houve decréscimo linear no rendimento com o aumento da população de plantas no espaçamento reduzido $(20 \mathrm{~cm})$. Estes resultados são conseqüencia do maior número de ramos, nós férteis e de legumes férteis. $\mathrm{m}^{-2}$ obtidos. $O$ arranjo com $20 \mathrm{~cm}$ de espaçamento e 20 plantas. $m^{-2}$ amenizou a competição intraespecifica, resultando em maior rendimento de grãos.
Palavras-chave: Glycine max, espaçamento, população, arranjo espacial.

\begin{abstract}
Plant arrangement that decrease interplant competition can improve soybean growth and yield by better use of resources. The experiment was performed at the Agronomic Experimental Station of the Universidade Federal do Rio Grande do Sul, in Eldorado do Sul, Rio Grande do Sul, Brazil, in the 2000/01 growing season. The objectives were to evaluate how soybean plant arrangement modify the interplant competition and the effect in grain yield and yield components. The cultivar tested was 'BRS $137^{\prime}$ (early, determinate), in no-till planting. The treatments were arranged in a split-plot randomized complete-block design,whith four replications. The treatments tested were two water availability (with and without irrigation), two row spacing (20 and $40 \mathrm{~cm})$ and three population levels $\left(20,30\right.$ and 40 plants. $\left.m^{-2}\right)$. The grain yield was affected by irrigation and by the interaction of row spacing and population. The irigated treatment $\left(5015 \mathrm{~kg} . \mathrm{ha}^{-1}\right)$ had grain yield $18 \%$ higher than the non irigated (4253kg. $\left.\mathrm{ha}^{-1}\right)$. The larger grain filling rate (GFR), resulted in larger seed weight, and explain, the greater yield in the irrigated treatment. The plant arrangemnt of 20 $\mathrm{cm}$ row spacing with the population of 20 plantas. $\mathrm{m}^{-2}$ provided the better results $\left(5014 \mathrm{~kg} . \mathrm{ha}^{-1}\right.$ compared to $4322 \mathrm{~kg} . \mathrm{ha}^{-1}$ in $40 \mathrm{~cm}$ row spacing). There is a linear decrease in grain yield with the increase in plant population with reduced row spacing $(20 \mathrm{~cm})$. These results can be explained by more branche number, fertil nodes and fertil pods. $m^{-2}$ obtained. The arrangement of $20 \mathrm{~cm}$ row spacing and 20 plants. $\mathrm{m}^{-2}$, attenuated the interplant competition resulting in larger grain yield.
\end{abstract}

${ }^{1}$ Engenheiro Agrônomo, MSc., Aluno de Doutorado da Faculdade de Agronomia, Universidade Federal do Rio Grande do Sul (FA/ UFRGS). Bolsista do CNPq. Departamento de Plantas de Lavoura. CP 776, 91501-970, Porto Alegre, RS. E-mail: lisandro@vortex.ufrgs.br Autor para correspondência.

${ }^{2}$ Engenheiro Agrônomo, PhD., Professor do Departamento de Plantas de Lavoura da FA/UFRGS. Bolsista do CNPq. E-mail: jame@.ufrgs.br

${ }^{3}$ Engenheiro Agrônomo, Doutor. E-mail: piresjl@vortex.ufrgs.br

${ }^{4}$ Engenheiro Agrônomo, MSc. E-mail: geovanoparcianello@vortex.ufrgs.br

${ }^{5}$ Aluno do Curso de Agronomia da UFRGS. Bolsista da FAPERGS. E-mail: ferreirafg@yahoo.com.br 
Key words: Glycine max, row spacing, plant population, plant arrangement.

\section{INTRODUÇÃO}

O arranjo de plantas pode ser modificado pela variação na população e pelo espaçamento entre linhas, alterando a área e a forma da área disponível para cada planta, o que se reflete numa competição intraespecífica diferenciada.

Trabalhos com população de plantas não têm mostrado efeito no rendimento de grãos, utilizando populações que variaram de 8 até 63 plantas.m ${ }^{-2}$ (RUBIN, 1997; PIRES et al.,1998). A inexistência de resposta diferenciada para rendimento de grãos à variação da população da soja está intimamente relacionada com a plasticidade fenotípica que esta cultura apresenta. Segundo COOPERATIVE... (1994), a plasticidade consiste na capacidade de a planta alterar sua morfologia e componentes do rendimento, a fim de adequá-los à condição imposta pelo arranjo de plantas.

CARPENTER \& BOARD (1997), em experimento com objetivo de determinar os mecanismos responsáveis pela compensação do rendimento por planta, para variações na população, concluíram que os ajustes no rendimento decorrentes de mudanças na população foram devido a alterações no número de legumes por planta. Esses ajustes resultaram de modificações da matéria seca dos ramos, que afetou o número de nós reprodutivos. O rendimento e matéria seca dos ramos por planta estavam altamente correlacionados $(r=0,95)$.

Em outros trabalhos, foi observada variação no rendimento com a modificação do número de plantas por área. MARQUES (1981), testando três populações de plantas $\left(25,35\right.$ e 45 plantas. $\left.m^{-2}\right)$, três espaçamentos entre linhas e quatro níveis de irrigação, obteve efeito significativo do fator população sobre o rendimento de grãos por área e número de legumes por planta, justificando tal resultado pela variação ocorrida no rendimento por planta e número final de plantas nas populações empregadas. HERBERT \& LITCHFIELD (1982), trabalhando com a variação na população de plantas e espaçamentos entre linhas, obtiveram aumento de $27 \%$ no rendimento com o aumento da população de plantas de 21 para 68 planta.m-2.

A redução do espaçamento entre linhas tem se constituído numa prática vantajosa, em que, na maioria dos experimentos, houve incremento do rendimento. Diversos trabalhos, utilizando espaçamentos entre linha de $17 \mathrm{~cm}$ até $100 \mathrm{~cm}$, têm verificado acréscimos de até $40 \%$ no rendimento
(HERBERT \& LITCHFIELD, 1982; ETHREDGE et al. 1989; BOARD et al., 1990; PIRES et al., 1998; VENTIMIGLIA et al., 1999) com a redução do espaçamento. Este aumento no rendimento tem sido associado a vários fatores, como o melhor uso da água devido ao sombreamento mais rápido do solo, melhor distribuição de raízes, redução da competição intraespecífica, maior habilidade de competição com plantas daninhas, exploração uniforme da fertilidade do solo e maior e mais rápida intercepcão da energia solar.

Poucos trabalhos foram realizados associando a redução do espaçamento entre linhas com a diminuição na população de plantas, razão pela qual o presente trabalho teve como objetivo avaliar como o arranjo de plantas da soja modifica a competição intraespecífica e de que forma isto se reflete no rendimento de grãos e seus componentes.

\section{MATERIAL E MÉTODOS}

O experimento foi conduzido a campo na Estação Experimental Agronômica da Universidade Federal do Rio Grande do Sul (EEA/UFRGS), localizada no município de Eldorado do Sul, região ecoclimática da Depressão Central do Estado do Rio Grande do Sul, em solo pertencente à unidade de mapeamento São Jerônimo, classificado como Argissolo Vermelho Distrófico típico (EMBRAPA, 1999). A recomendação de adubação, baseada na análise de solo, indicou a quantidade de $50 \mathrm{~kg} \cdot \mathrm{ha}^{-1}$ de $\mathrm{P}_{2} \mathrm{O}_{5}$ e $50 \mathrm{~kg}$.ha ${ }^{-1}$ de $\mathrm{K}_{2} \mathrm{O}$. Para suprir essa necessidade, foram aplicados $250 \mathrm{~kg}$.ha ${ }^{-1}$ de adubo químico da fórmula 5-20-20.

O delineamento experimental utilizado foi o de blocos ao acaso com parcelas sub-subdivididas e quatro repetições. Os tratamentos constaram de dois regimes hídricos (irrigado e não irrigado), nas parcelas principais; dois espaçamentos entre linhas (20 e 40cm), nas subparcelas, e três populações de plantas $(20,30$ e 40 plantas. $\mathrm{m}^{-2}$ ) nas sub-subparcelas.

Utilizou-se a cultivar 'BRS 137', de ciclo semi-precoce e hábito de crescimento determinado. $\mathrm{O}$ experimento foi instalado em semeadura direta, em solo com cobertura de $5800 \mathrm{~kg} \cdot \mathrm{ha}^{-1}$ de aveia preta mais ervilhaca no início do experimento. As sementes foram tratadas com fungicida [captan $(90 \mathrm{~g}$ de i.a. por $100 \mathrm{~kg}$ de semente)] e inoculadas com Bradyrhizobium japonicum em meio turfoso (400 $\mathrm{g}$ de inoculante por $50 \mathrm{~kg}$ de semente). A semeadura foi realizada em 15/11/ 2000, sendo essa época recomendada preferencial para a região, utilizando semeadora de parcelas. Aos 15 dias após emergência, quando as plantas estavam no estádio V2 (COSTA \& MARCHEZAN, 1982) realizou- 
se o desbaste, ajustando-se para as populações desejadas. Manteve-se o experimento livre de pragas e plantas daninhas.

A umidade do solo foi monitorada com tensiômetros, realizando-se a irrigação por aspersão quando a tensão da água ultrapassava o limite de $-0,05$ MPa.

O rendimento de grãos $\left(\mathrm{kg} \cdot \mathrm{ha}^{-1}\right)$ foi obtido pela colheita de 2 e 4 linhas centrais de cada subsubparcela, nos espaçamentos de 40 e $20 \mathrm{~cm}$ entre linhas, respectivamente. Descontaram-se as bordaduras de $50 \mathrm{~cm}$ em cada extremidade de linhas. O peso de grãos foi corrigido para $13 \%$ de umidade e para um hectare.

As determinações do número de legumes e de nós férteis e número de ramos em $\mathrm{R} 8$, foram efetuadas pela contagem destes componentes em 10 plantas da área útil e os valores expressos por $\mathrm{m}^{2}$. O peso de 100 grãos foi obtido pela pesagem de quatro amostras de 100 grãos coletadas aleatoriamente dos grãos colhidos na área útil de cada sub-subparcela e corrigidos para $13 \%$ de umidade. O número de grãos por legume fértil foi calculado dividindo-se o número de grãos pela quantidade de legumes férteis encontrados por planta. Para o cálculo da taxa de enchimento de grãos (TEG), foram utilizados os dados obtidos no período reprodutivo, aplicados a fórmula proposta por COSTA et al. (1991).

Os resultados obtidos foram submetidos à análise de variância, pelo teste $\mathrm{F}$, sendo a diferença entre médias de tratamentos comparadas pelo teste de Duncan a $5 \%$ de probabilidade de erro para os fatores irrigação e espaçamento entre linhas. O fator população de plantas foi comparado por análise de regressão.

\section{RESULTADOS E DISCUSSÃO}

A irrigação, neste trabalho, tinha como objetivo criar dois ambientes diferenciados. $\mathrm{O}$ ano agrícola apresentou condições de precipitação favoráveis durante quase todo o ciclo da soja. Ocorreu deficiência hídrica somente no final do ciclo (estádio R6 - máximo volume de grãos), que foi amenizada por três irrigações. Mesmo tendo-se irrigado somente nesta fase, o tratamento irrigado $\left(5015 \mathrm{~kg} \cdot \mathrm{ha}^{-1}\right)$ apresentou rendimento de grãos $18 \%$ superior ao não irrigado (4253kg.ha-1 ${ }^{-1}$. Esta resposta ocorreu em função da maior TEG do tratamento irrigado, que resultou em grãos mais pesados, refletindo-se em maior rendimento de grãos (Tabela 1). Os demais componentes do rendimento (Tabela 1) não foram afetados pelo regime hídrico por ter-se aplicado o tratamento irrigado somente no estádio R6 quando esses já estavam praticamente definidos.

Estes resultados podem ser explicados pelo fato de que a deficiência hídrica, durante o enchimento de grãos, reduz o tamanho e peso do grão, devido à diminuição do suprimento de fotoassimilados pela planta e/ou inibição do metabolismo do grão (SALINAS et al., 1996). Pode ocorrer também a redução no rendimento pela diminuição da atividade fotossintética da folha e pela menor remobilização de C e N para o grão (SOUZA et al., 1997).

Resultados de outros pesquisadores como os obtidos por DESCLAUX et al. (2000) também constataram redução no peso do grão em função do estresse hídrico, ocorrido no período de enchimento de grãos da soja. Esse estresse pode ser amenizado pela irrigação, o que é confirmado em trabalhos em que a irrigação aumentou o peso do grão (THOMAS \& COSTA, 1994; MAEHLER, 2000).

Houve resposta do rendimento de grãos para a interação de espaçamento entre linhas e população de plantas (Tabela 2). O arranjo de plantas que apresentou maior rendimento foi a combinação da população de 20 plantas. $\mathrm{m}^{-2}$ com o espaçamento de $20 \mathrm{~cm}\left(5014 \mathrm{~kg} \cdot \mathrm{ha}^{-1}\right)$ em comparação ao espaçamento de $40 \mathrm{~cm}\left(4322 \mathrm{~kg} \cdot \mathrm{ha}^{-1}\right)$ nesta mesma população. O maior rendimento obtido neste arranjo está relacionado com

Tabela 1 - Rendimento de grãos, componentes do rendimento e taxa de enchimento de grãos (TEG) da cultivar de soja BRS 137 em dois regimes hídricos, na média de dois espaçamentos entre linhas e três populações de plantas. EEA/UFRGS, Eldorado do Sul, RS, 2000/01

\begin{tabular}{|c|c|c|c|c|}
\hline Regime hídrico & Irrigado & Não irrigado & Média & C.V. $(\%)$ \\
\hline Rendimento de grãos (kg.ha $\left.{ }^{-1}\right)$ & $5015 \mathrm{a}^{*}$ & $4253 \mathrm{~b}$ & 4634 & 6,5 \\
\hline Legumes férteis.m ${ }^{-2}$ & $984 \mathrm{a}$ & $934 \mathrm{a}$ & 959 & 3,3 \\
\hline Grãos/legume & $2,20 \mathrm{a}$ & $2,20 \mathrm{a}$ & 2,20 & 1,0 \\
\hline Peso de 100 grãos & $19,0 \mathrm{a}$ & $15,6 \mathrm{~b}$ & 17,3 & 2,6 \\
\hline TEG $\left(\mathrm{g} \cdot \mathrm{m}^{-2} \cdot \mathrm{dia}^{-1}\right)$ & $13,5 \mathrm{a}$ & $11,9 \mathrm{~b}$ & 12,7 & 5,1 \\
\hline
\end{tabular}

*Médias não seguidas de mesma letra, na linha, diferem pelo teste de Duncan ao nível de 5\% de probabilidade de erro.

Ciência Rural, v. 33, n. 3, mai-jun, 2003. 
Tabela 2 - Rendimento de grãos e características agronômicas da cultivar de soja BRS 137 sob diferentes arranjos de plantas na média de dois regimes hídricos. EE A/UFRGS, Eldorado do Sul, RS, 2000/01.

\begin{tabular}{|c|c|c|c|c|c|c|c|c|}
\hline População (plantas.m ${ }^{-2}$ ) & \multicolumn{2}{|c|}{20} & \multicolumn{2}{|c|}{30} & \multicolumn{2}{|c|}{40} & \multirow{2}{*}{ Média } & \multirow{2}{*}{$\begin{array}{c}\text { CV (\%) } \\
\text { Espaçamento }\end{array}$} \\
\hline Espaçamento $(\mathrm{cm})$ & 20 & 40 & 20 & 40 & 20 & 40 & & \\
\hline Rendimento (kg.ha ${ }^{-1}$ ) & $5014 \mathrm{a}^{*}$ & $4322 \mathrm{~b}$ & $4581 \mathrm{a}$ & $4581 \mathrm{a}$ & $4686 \mathrm{a}$ & $4618 \mathrm{a}$ & 4634 & 6,5 \\
\hline $\mathrm{N}^{\mathrm{o}}$ de ramos. $\mathrm{m}^{-2}$ & $150 \mathrm{a}$ & $92 \mathrm{~b}$ & 94 a & $90 \mathrm{a}$ & $110 \mathrm{a}$ & $99 \mathrm{~b}$ & 106 & 5,9 \\
\hline Nós férteis.m ${ }^{-2}$ & $67 \mathrm{a}$ & $41 \mathrm{~b}$ & $42 \mathrm{a}$ & $40 \mathrm{a}$ & $49 \mathrm{a}$ & $44 \mathrm{~b}$ & 48 & 6,3 \\
\hline Legumes férteis. $\mathrm{m}^{-2}$ & 1329 a & $837 \mathrm{~b}$ & $850 \mathrm{a}$ & $844 \mathrm{a}$ & $986 \mathrm{a}$ & 909 a & 959 & 7,8 \\
\hline Grãos/legume & $2,20 \mathrm{a}$ & $2,20 \mathrm{a}$ & $2,19 \mathrm{a}$ & $2,19 \mathrm{a}$ & $2,21 \mathrm{a}$ & $2,20 \mathrm{a}$ & 2,20 & 1,9 \\
\hline Peso 100 grãos (g) & $18,5 \mathrm{a}$ & $16,4 \mathrm{~b}$ & $17,4 \mathrm{a}$ & $16,8 \mathrm{a}$ & $17,4 \mathrm{a}$ & $17,1 \mathrm{a}$ & 17,3 & 2,7 \\
\hline TEG $\left(\mathrm{g} \cdot \mathrm{m}^{-2} \cdot \mathrm{dia}^{-1}\right)$ & $15,7 \mathrm{a}$ & $10,7 \mathrm{~b}$ & $12,7 \mathrm{a}$ & $11,4 \mathrm{a}$ & $13,7 \mathrm{a}$ & $12,1 \mathrm{~b}$ & 12,7 & 15,0 \\
\hline
\end{tabular}

Médias não seguidas de mesma letra, na linha (para cada população), diferem pelo teste de Duncan ao nível de 5\% de probabilidade de erro.

a diminuição da competição intraespecífica. Segundo PIRES et al. (1998), a redução na competição ocorre, principalmente, por luz, mas também permite melhor aproveitamento de água, interceptação mais rápida da radiação e maior exploração do solo pelas raízes. Nas demais populações, não houve resposta para o espaçamento entre linhas.

Considerando a competição entre plantas como um fator isolado, era de se esperar melhores resultados com o arranjo de 30 plantas. $\mathrm{m}^{-2} \mathrm{com} 20 \mathrm{~cm}$ de espaçamento entre linhas, pois este se aproxima mais da eqüidistância. No entanto, as condições meteorológicas favoráveis, ocorridas no ano, principalmente em relação à pequena deficiência hídrica, podem ter favorecido a combinação da menor população de plantas com o menor espaçamento entre linhas.

MAEHLER (2000), por sua vez, não obteve aumento no rendimento com a redução do espaçamento entre linhas de $40 \mathrm{~cm}$ para $20 \mathrm{~cm}$, utilizado a população de 40 plantas. $\mathrm{m}^{-2}$. Conforme salientado pelo autor, a redução do espaçamento entre linhas pode não trazer benefícios em rendimento, mas também não o reduz, fato importante já que esta prática não aumenta os custos de produção da lavoura.

No espaçamento reduzido $(20 \mathrm{~cm})$, verificouse diminuição linear no rendimento de grãos com o aumento da população de plantas (Figura 1a). ETHREDGE et al. (1989) obtiveram redução linear no rendimento de grãos do caule e dos ramos das plantas de soja com o aumento da população de plantas. Essa diminuição pode não acarretar diminuição no rendimento por área, pois o maior número de plantas compensaria o menor rendimento por planta, o que não foi observado neste trabalho.

Os rendimentos obtidos, com a modificação do arranjo de plantas, devem-se a modificações morfofisiológicas, podendo ser melhor entendidas com a análise dos componentes do rendimento e da morfologia da planta. A redução do espaçamento entre linhas aumentou o número de ramos, em relação a $40 \mathrm{~cm}$, nas populações de 20 e 30 plantas. $m^{-2}$ (Tabela 2). O número de ramos apresentou diminuição linear com o aumento da população de plantas como reflexo da competição entre essas (Figura 1b). BOARD et al. (1990) afirmam que, para uma mesma população de plantas, a diminuição do espaçamento entre linhas da soja aumenta o número e o comprimento dos ramos.

Segundo BALLARÉ et al. (1995), o crescimento das plantas é modificado pela população de plantas, sendo que isto ocorre, em parte, por mecanismos que usam informações sobre a luz do ambiente, por meio de fotosensores específicos. Exemplificam que, com o aumento da população, ocorrem mudanças na relação vermelho extremo/ vermelho, que atuarão como sinais para que a planta diminua o número de ramos.

O número de ramificações por planta da soja e seu desenvolvimento está correlacionado com a competição intraespecífica por fatores do meio como água, luz e nutrientes (THOMAS et al., 1998). PARVEZ et al. (1989) observaram que, para um mesmo espaçamento entre linhas, a diminuição da população de plantas de soja de 40 para 20 e 10 plantas. $\mathrm{m}^{-2}$, aumentou o número e o comprimento total de ramos.

O maior número de ramos observado, em alguns tratamentos, pode ter sido responsável pelo aumento linear no número de nós férteis, com a diminuição da população de plantas dentro de $20 \mathrm{~cm}$ de espaçamento (Figura 1c), bem como o maior número destes obtido nas populações de 20 e 30 plantas. $\mathrm{m}^{-2}$, combinado com $20 \mathrm{~cm}$ em comparação a $40 \mathrm{~cm}$ (Tabela 2).

Neste trabalho, o maior número de ramos, obtido nos tratamentos com maior rendimento, pode ter proporcionado maior número de locais para formação de estruturas reprodutivas, resultando em maior número de legumes férteis (Tabela 2 e Figura 1d) e contribuindo para o aumento no rendimento. 


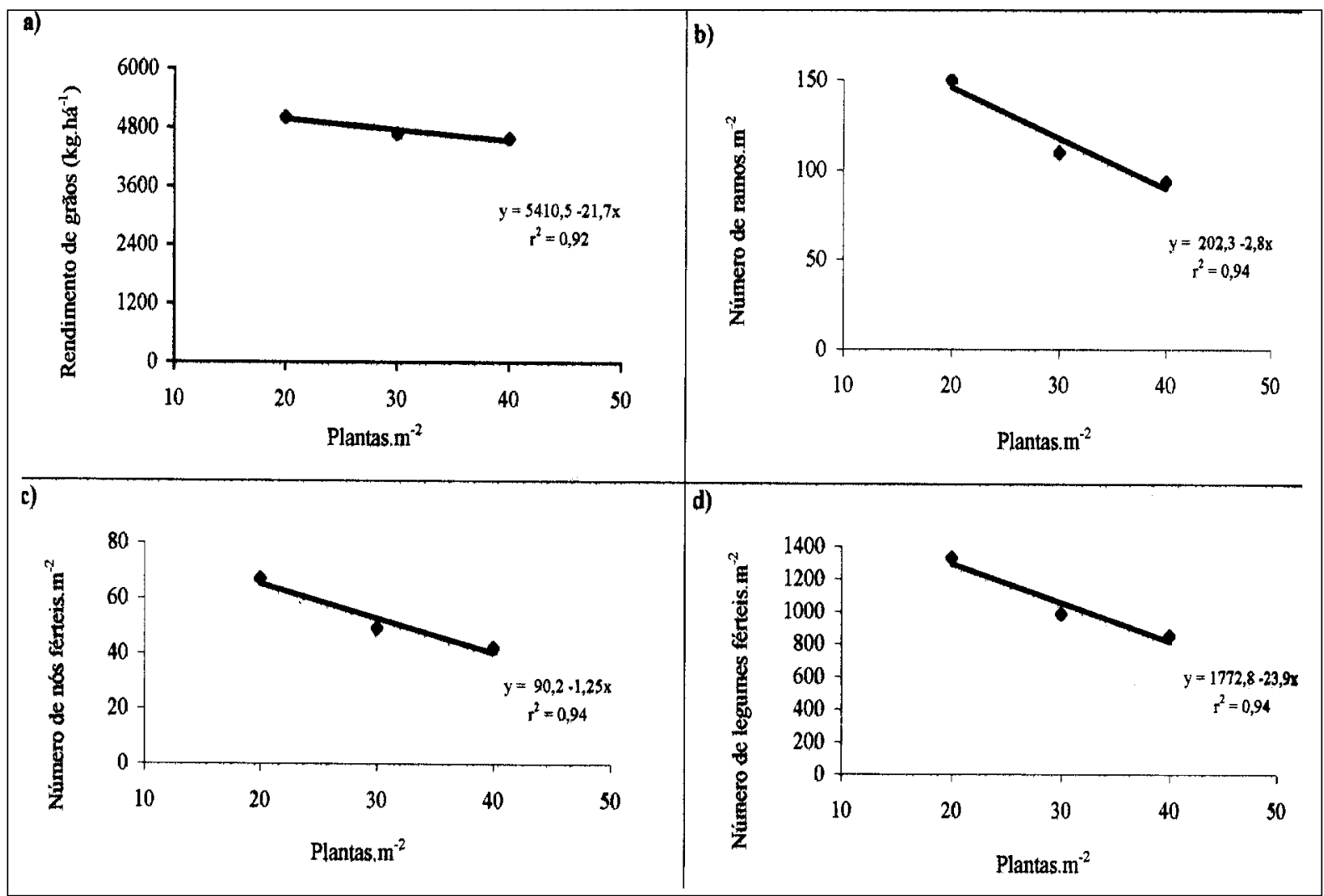

Figura 1 - Rendimento de grãos (a), número de ramos (b), número de nós férteis (c) e número de legumes férteis (d), no espaçamento de $20 \mathrm{~cm}$ entre linhas, em função DA população de plantas, NA média de dois regimes hídricos. EEA/UFRGS, Eldorado do Sul, RS, $2000 / 01$.

CARPENTER \& BOARD (1997) concluíram que os ajustes no rendimento por planta, decorrentes de mudanças na população, foram devido a alterações no número de legumes nos ramos. Estes ajustes resultaram de alterações da matéria seca dos ramos por planta, o que afetou o número de nós reprodutivos dos ramos. O número de legumes é o componente do rendimento que mais sofre modificações pela utilização de práticas de manejo diferenciadas (COOPERATIVE..., 1994). THOMAS (1992) afirma que os legumes produzidos nos ramos da planta de soja contribuem com até $70 \%$ do rendimento de grãos.

A maior demanda de fotoassimilados, proporcionada pelo maior número de legumes, pode ter sido suprida nos tratamentos com menor competição intraespecífica, principalmente por luz. O dossel da soja se caracteriza por apresentar uma camada superior de folhas, que dificulta a penetração de luz nos estratos inferiores. BERGAMASCHI et al. (1981), medindo a penetração de luz no perfil do dossel, verificaram que, no início do período reprodutivo, cerca de $50 \%$ da radiação líquida estava disponível no nível do solo. Nos estádios R5 (início do enchimento de grãos) e R6, apenas $10 \%$ desta radiação atingia à parte inferior da comunidade de plantas e $20 \%$ à parte média.

Os arranjos utilizados neste trabalho, que proporcionaram melhor distribuição das plantas na área, podem ter aumentado a penetração de luz nos estratos inferiores do dossel, aumentando a produção fotossintética, contribuindo com o aumento no rendimento de grãos. PIRES et al. (1998), em trabalho objetivando avaliar os efeitos do arranjo de plantas e níveis de adubação sobre os componentes do rendimento e rendimento de grãos da soja, comentam que, embora não tenha sido determinado naquele trabalho características relacionadas com a penetração de luz na comunidade de plantas, ficou evidente a ocorrência de melhor captação de luz.

O número de grãos por legume não sofreu alterações com a modificação do arranjo de plantas (Tabela 2). BOARD et al. (1990) observaram que o tamanho do grão e o número de grãos por legume não foram afetados pelo arranjo de plantas, explicando que isto ocorreu em função destes componentes serem

Ciência Rural, v. 33, n. 3, mai-jun, 2003. 
determinados no final do ciclo reprodutivo da soja. Tanto o número de grãos por legume como o peso do grão tem controle genético substancial e por isso tem pequena variação (COOPERATIVE..., 1994). Contrariamente a esta afirmação e aos resultados obtidos por alguns autores (PIRES et al., 1998; THOMAS et al., 1998; MAEHLER, 2000), o peso do grão (expresso pelo peso de 100 grãos) variou em função da interação do fator espaçamento entre linhas e população de plantas (Tabela 2). Alguns trabalhos mostram que o peso do grão é alterado pelo arranjo de plantas. MOORE (1991) observou que o peso e o tamanho dos grãos aumentaram quando o espaçamento entre plantas era eqüidistante, e que esse aumento ocorreu também com a diminuição da população.

As respostas obtidas, quanto ao peso do grão, neste trabalho, estão relacionadas com a TEG (Tabela 2), que apresentou resultados similares aos obtidos nesta variável. PIRES (1998) observou uma taxa média de enchimento de grãos $25 \%$ superior em espaçamento entre linhas reduzido $(20 \mathrm{~cm})$, comparado com $40 \mathrm{~cm}$. Já MAEHLER (2000) não obteve diferenças significativas, em experimento utilizando estes dois espaçamentos entre linhas, no que se refere a esta variável.

Os resultados obtidos, nos arranjos com maior rendimento de grãos, devem-se ao maior número de ramos, de nós férteis e de legumes férteis; à TEG e peso do grão superiores. Estas respostas foram obtidas sob condições meteorológicas favoráveis ao crescimento da soja, principalmente em relação à precipitação, havendo a necessidade de se realizar também este trabalho sob condições ambientais adversas.

\section{CONCLUSÕES}

O estresse hídrico afeta o rendimento da soja mesmo quando ocorre no final do ciclo, principalmente pela diminuição do peso do grão;

O arranjo de plantas com menor espaçamento e população de plantas proporciona menor competição intraespecífica, resultando em maior rendimento de grãos, ocasionado pelo maior número de legumes férteis $/ \mathrm{m}^{2}$ associado ao maior peso do grão.

\section{REFERÊNCIAS BIBLIOGRÁFICAS}

BALLARÉ, C.L.; SCOPEL, A.L.; SÁNCHEZ, R.A. Plant photomorphogenesis in canopies, crop growh, and yield. Hort Science, St. Joseph, v.30, n.6, p.1172-1181, 1995.
BERGAMASCHI, H. et al. Perfis de radiação em uma comunidade de soja (Glycine $\max$ (L.) Merril), em dois estadios de desenvolvimento. In: CONGRESSO BRASILEIRO DE AGrometeorologia, 2, 1981, Pelotas. Anais... Pelotas: Sociedade Brasileira de Agrometeorologia, 1981. p. 173-178.

BOARD, J.E.; HARVILLE, B.G.; SAXTON, A.M. Narrowrow seed-yield enhancement indeterminate soybean. Agronomy Journal, Madison, v.82, n.1, p.64-68, 1990.

CARPENTER, A.C.; BOARD, J.E. Growth dynamic factors controlling soybean yield stability across plant populations. Crop Science, Madison, v.37, n.5, p.1520-1526, 1997.

COOPERATIVE EXTENSION SERVICE AMES. How a soybean plant develops. Ames : Iowa State University of Science and Technology, 1994. 20p.

COSTA, J.A.; MARCHEZAN, E. Características dos estádios de desenvolvimento da soja. Campinas : Fundação Cargil, 1982. 30p.

COSTA, J.A.; TEIXEIRA, M.C.C.; MARCHEZAN, E. Taxa e duração do acúmulo de matéria seca nos grãos de soja e sua relação com o rendimento. Pesquisa Agropecuária Brasileira, Brasília, v.26, n.9, p.1577-1582, 1991.

DESCLAUX, D.; HUYNH, T.; ROUMET, P. Identification of soybean plant characteristics that indicate the timing of drought stress. Crop Science, Madison, v.40, n.3, p.716722,2000 .

EMBRAPA. Centro Nacional de Pesquisas de Solos. Sistema Brasileiro de Classificação de Solos. Brasília : EMBRAPA, 1999. 412p.

ETHREDGE, W.J.; ASHLEY, D.A.; WOODRUFF, J. M. Row spacing and plant population effects on yield components of soybean. Agronomy Journal, Madison, v.81, n.6, p.947$951,1989$.

HERBERT, S.J.; LITCHFIELD, G.V. Partitioning soybean seed yield components. Crop Science, Madison, v.22, n.5, p.10741079, 1982.

MAEHLER, A.R. Crescimento e rendimento de duas cultivares de soja em resposta ao arranjo de plantas e regime hídrico. 2000. 108f. Dissertação (Mestrado em Fitotecnia) - Faculdade de Agronomia, Universidade do Rio Grande do Sul, Porto Alegre.

MARQUES, J. B. B. Efeito do espaçamento entre fileiras, população de plantas e irrigação sobre o rendimento da planta, rendimento e qualidade da semente da soja (Glycine max (L.) Merril). 1981. 93f. Dissertação (Mestrado em Fitotecnia) - Faculdade de Agronomia, Universidade do Rio Grande do Sul, Porto Alegre.

MOORE, S.H. Uniformity of planting spacing effect on soybean population parameters. Crop Science, Madison, v.31, n.4, p.1049-1051, 1991.

PARVEZ, A.Q.; GARDNER, F. P.; BOOTE, K. J. Determinate and inderterminate type soybean cultivar response to patterns, density and planting date. Crop Science, Madison, v.29., n.1, p.150-157, 1989. 
PIRES, J. L. Efeito da redução do espaçamento entre linhas da soja sobre o rendimento de grãos e seus componentes, em semeadura direta. 1998. 94f. Dissertação (Mestrado em Fitotecnia) - Faculdade de Agronomia, Universidade do Rio Grande do Sul, Porto Alegre.

PIRES, J.L.; COSTA, J.A.; THOMAS, A. L. Rendimento de grãos de soja influenciado pelo arranjo de plantas e níveis de adubação. Pesquisa Agropecuária Gaúcha, Porto Alegre, v.4, n.2, p.183-188, 1998

RUBIN, S. de A.L. Comportamento da cultivar "FEPAGRORS 10" em seis densidades de semeadura ano planalto médio riograndense. In: REUNIÃO DE PESQUISA DE SOJA DA REGIÃo SUL, 25, 1997, Passo Fundo, RS. Anais... Passo Fundo : EMBRAPA, 1997. p.187.

SALINAS, A.R. et al. Respuestas fisiológicas que caracterizan el comportamiento de diferentes cultivares de soja a la deficiência hídrica. Pesquisa Agropecuária Brasileira, Brasília, v.31, n.5, p.331-338, 1996.

SOUZA, P.I.; EGLI, D.B.; BRUENING, W. Water stress during seed filling and leaf senescence in soybean. Agronomy Journal, Madison, v.89, n.5, p.807-812, 1997.

THOMAS, A.L. Desenvolvimento e rendimento da soja em resposta à cobertura morta e à incorporação de gesso ao solo, com e sem irrigação. 1992. 91f. Dissertação (Mestrado em Fitotecnia) - Faculdade de Agronomia, Universidade Federal do Rio Grande do Sul, Porto Alegre.

THOMAS, A.L.; COSTA, J.A. Influência do déficit hídrico sobre o desenvolvimento e rendimento da soja. Pesquisa Agropecuária Brasileira, Brasília, v.29, n.9, p.1389-1396, 1994.

THOMAS, A.L.; COSTA, J.A.; PIRES, J.L. Rendimento de grãos de soja afetado pelo espaçamento entre linhas e fertilidade do solo. Pesquisa Agropecuária Brasileira, Brasília, v.28, n.4, p.543-546, 1998.

VENTIMIGLIA, L.A. et al. Potencial de rendimento da soja em razão da disponibilidade de fósforo no solo e dos espaçamentos. Pesquisa Agropecuária Brasileira, Brasília, v.34, n.2, p.195-199,1999. 